\title{
Tetrahydroxystilbene Glucoside Protects Against Oxidized LDL-Induced Endothelial Dysfunction via Regulating Vimentin Cytoskeleton and its Colocalization with ICAM-1 and VCAM-1
}

\author{
Wenjuan Yao Chao Huang Qinju Sun Xiang Jing Huiming Wang Wei Zhang \\ Department of Pharmacology, School of Pharmacy, Nantong University, Nantong, China
}

\section{Key Words}

Tetrahydroxystilbene glucoside • Oxidized LDL • Endothelial dysfunction • Vimentin

\begin{abstract}
Background: Endothelial cell dysfunction triggered by oxidized low-density lipoprotein (oxLDL) is the main event occurring during the development of atherosclerosis. 2,3,5,4'-tetrahydroxystilbene-2-O- $\beta$-D-glucoside (TSG), an active component of the rhizome extract from Polygonum multiflorum, exhibits significant anti-atherosclerotic activity. However, the protective effects of TSG against oxLDL-induced endothelial dysfunction have not been clarified. We investigated the cytoprotective effects of TSG in human umbilical vein endothelial cells (HUVECs) and explored underlying mechanisms. Methods and Results: TSG pretreatment markedly attenuated oxLDL-mediated loss of cell viability, release of lactate dehydrogenase $(\mathrm{LDH})$, cell apoptosis, and monocyte adhesion. OxLDL increased vimentin mRNA and protein levels, vimentin cleavage, caspase- 3 activation, adhesion molecules levels and their colocalization with vimentin in HUVECs. These alterations were attenuated by pretreatment with TSG. Meanwhile, TSG inhibited both the expression of TGF $\beta 1$ and the phosphorylation of Smad2 and Smad3, and TSG suppressed the nuclear translocation of Smad4 induced by oxLDL. Using shRNA, oxLDL-induced cell apoptosis and monocyte adhesion were significantly inhibited by vimentin suppression in HUVECs. Conclusions: These results suggest that TSG protects HUVECs against oxLDL-induced endothelial dysfunction through inhibiting vimentin expression and cleavage, and the expression of adhesion molecules and their colocalization with vimentin. The interruption of TGF $\beta /$ Smad pathway and caspase- 3 activation appears to be responsible for the downregulation of TSG on vimentin expression and fragmentation, respectively.
\end{abstract}




\section{Introduction}

Endothelial dysfunction is a driving force in the initiation and development of atherosclerosis [1]. The risk factors for endothelium injury include oxidized low-density lipoprotein (oxLDL), Angiotensin II, advanced glycosylation end products (AGEs), and sheer stress, etc., among which the ox-LDL is now considered to play a crucial role [2]. It has been reported that ox-LDL induces the toxic effects or activation in endothelial cells, including an increase in reactive oxygen species (ROS) production, cell apoptosis and expression of adhesion molecules that facilitate the firm adhesion and activation of leukocytes and platelets [3-5].

$2,3,5,4^{\prime}$-Tetrahydroxystilbene-2-0- $\beta$-D-glucoside (TSG; Fig. $1 \mathrm{~A}$ ) is an active component of the rhizome extract with polyphenolic structure from the famous traditional Chinese herb Polygonum multiflorum. TSG has received a great deal of attention owing to its biological properties, including antioxidative and anti-inflammatory effects [6, 7]. TSG has been shown in various studies to inhibit matrix metalloproteinases activation, inflammation and vascular endothelial dysfunction in atherosclerotic rats $[8,9]$; reduce proliferation of vascular smooth muscle cells, oxidation of lipoprotein, vascular senescence and endothelial senescence [1013]. However, whether TSG can inhibit oxLDL-induced experimental endothelial dysfunction remains unknown.

Our recent studies have shown that vimentin is one of the key proteins responsible for atherogenesis suppression induced by TSG treatment [14]. Vimentin is a member of the intermediate filament protein family and has been shown to be present in cells of the blood vessel walls. Vimentin has been reported to be involved in proliferation of VSMC, migration of monocytes across the endothelium walls and formation of foam-cells [15-17]. It has been demonstrated that vimentin expression is required for cell adhesion and migration [18-21]. Recent studies suggested that vimentin regulated both the expression and the distribution of adhesion molecules which were important for the transmigration process, such as intercellular adhesion molecules 1 (ICAM-1) and vascular cell adhesion molecule-1 (VCAM1) $[16,22]$. Moreover, many investigations have shown that vimentin structure was rapidly proteolyzed by different caspases into multiple fragments during apoptosis induced by many stimuli [23-27]. Vimentin expression is often regulated in response to TGF $\beta$ in many cell types. Vimentin mRNA was reported to be stimulated by TGF $\beta 1$ via the canonical Smaddependent signaling in C2C12 myoblastic cells [28]. Nevertheless, whether TSG can regulate vimentin structure in oxLDL-induced endothelial dysfunction and the exact molecular mechanisms of this regulation have not yet been fully elucidated.

Herein, we investigated the protective effects of TSG on oxLDL-induced endothelial dysfunction, and further demonstrated TSG regulation on vimentin cytoskeleton and the colocalization of vimentin and adhesion molecules.

\section{Materials and Methods}

\section{Chemicals}

TSG (purity above 98\%) was obtained from Sikehua Biosciences (\#2005119; Chengdu, China). Medium 199 (M199), Roswell Park Memorial Institute (RPMI) 1640 and fetal bovine serum (FBS) were obtained from GibcoBRL (Grand Island, NY). 3-(4, 5-Dimethylthiazol-2-yl)-2, 5-diphenyltetrazolium bromide (MTT), Hoechst 33258, and trypsin were obtained from Sigma-Aldrich (St. Louis, MO, USA). The reagent kit for determining lactate dehydrogenase (LDH) was purchased from Nanjing Jiancheng Institute of Biological Engineering (Nanjing, China). Terminal deoxynucleotidyl transferase-mediated dUTP nick end labeling (TUNEL) assay kit and caspase-3 activity assay kit were from BiYuntian Biological Technology Institution (Shanghai, China). Rabbit anti-vimentin, anti-TGF- $\beta 1$, anti-phospho-Smads (-2, -3), anti-Smad4, anti-GAPDH, anit-caspase3, anti-ICAM-1 and anti-VCAM-1 antibodies were purchased from Cell Signaling Technology (Beverly, MA, USA). Mouse monoclonal anti-vimentin and FITC-conjugated anti-mouse IgG antibodies were purchased from Boster Company (Wuhan, China). Horseradish peroxidase (HRP)- and Cy3-conjugated 
Yao et al.: TSG Inhibits Vimentin and Adhesion Molecules

anti-rabbit IgG were purchased from Santa Cruz Biotechnology (Santa Cruz, CA, USA). The primers were synthesized by Sangon Gene Company (Shanghai, China).

\section{Cell culture, LDL isolation and oxidation}

Human umbilical vein endothelial cells (HUVECs) were isolated by collagenase digestion of umbilical veins from fresh cords. The study protocol was approved by the Ethics Review Committee of the Affiliated Hospital of Nantong University. HUVECs were cultured in M199 containing 20\% FBS until the monolayer became confluent. The medium was then changed to M199 with 2\% FBS and refreshed every three days. HUVECs at passages 3-6 were used in the present study and identified by cobblestone morphology and strong positive immunoreactivity to von Willebrand factor (data not shown). U937, a human histiocytic leukemia cell line, was obtained from ATCC (Rockville, MD) and cultured in RPMI1640 with 10\% FBS.

Human native LDL was isolated from human plasma by sequential ultracentrifugation using a previously described method [29]. For oxidation, LDL was incubated with $10 \mu \mathrm{M} \mathrm{CuSO}_{4}$ as the oxidant for $24 \mathrm{~h}$ at room temperature [5]. The extent of oxidation was determined by thiobarbituric acid-reacting substances (TBARS) assay.

\section{Cell viability and $L D H$ release assay}

HUVECs were pretreated for $12 \mathrm{~h}$ with various doses $(1,10,25,50$, and $100 \mu \mathrm{M}$, respectively) of TSG. After removing the supernatant, the cells were exposed to $200 \mu \mathrm{g} / \mathrm{ml}$ of oxLDL for an additional $24 \mathrm{~h}$. Cell viability was determined by MTT assay as described [30]. The viability of the group not exposed to either oxLDL or TSG was set to $100 \%$, and that of the other groups was expressed as the percentage of control cells. Cell injury was confirmed further by measuring the activity of LDH that was released from the damaged cells into the culture medium. LDH activity in the medium was determined according to the protocols of an LDH kit.

\section{Analysis of apoptotic cells and adhesion assay}

The nuclear chromatin morphological changes of apoptotic cells were analyzed by nuclear staining with Hoechst 33258. Briefly, HUVECs were fixed with $70 \%$ ethanol for $30 \mathrm{~min}$ at $37^{\circ} \mathrm{C}$ and then stained with Hoechst $33258(1 \mu \mathrm{g} / \mathrm{ml}$ in PBS) for $10 \mathrm{~min}$. Apoptotic cells were stained bright blue due to their condensed or fragmented nuclei. In addition, apoptotic cells were assessed by TUNEL assay according to the manufacturer's protocol. The images of Hoechst- and TUNEL-positive cells were visualized by fluorescence microscopy (Nikon, Japan). Cells were counted in four random fields in each of three different slides, and the apoptosis rate was quantified by the percentage of positive cells.

Adhesion of U937 monocytes to HUVECs was quantified by microscopy as previously described [31, 32].

Western blot analysis and caspase-3 activity assay

HUVECs with various treatments were lysed in protein lysis buffer (0.2\% SDS, 1\% NP-40, 5 mM EDTA, $1 \mathrm{mM}$ PMSF, $10 \mu \mathrm{g} / \mathrm{mL}$ leupeptin, and $10 \mu \mathrm{g} / \mathrm{mL}$ aprotinin). Cell lysates (15-30mg protein) were separated by $12 \%$ sodium dodecyl sulfate-polyacrylamide gel electrophoresis (SDS-PAGE), and then transferred to a polyvinylidene fluoride (PVDF) membrane (Millipore, Billerica, MA, USA). Blots were incubated with specific antibodies and then with HRP-conjugated secondary antibodies. Band intensity was quantified by Densitometry and Imaging Software (Labworks). Total protein expression was normalized to GAPDH levels.

The activity of caspase- 3 was measured according to the kit manufacturers' instructions. HUVECs were treated for $15 \mathrm{~min}$ with iced lysis buffer supplied with the kit. Then the supernatants were treated for 100 $\min$ at $37^{\circ} \mathrm{C}$ with Ac-DEVD-p-NA (acetyl-Asp-Glu-Val-Asp-pnitroaniline) which was cleaved by caspase-3 to form p-NA. Caspase-3 activity was read in a microtiter plate reader at $405 \mathrm{~nm}$.

\section{Quantitative PCR (qRT-PCR)}

Total RNA from HUVECs wasisolated by Trizol reagent(Invitrogen) and wasusedfor reverse transcription with a reverse transcript synthesis kit (TaKaRa, Dalian, China). The obtained cDNA was then mixed with Maxima SYBR Green qPCR Master Mix (Fermentas, Burlington, Canada) and primers (Sangon, Shanghai, China). The primer sequences were as follows: vimentin, (F) 5'-GCCTATGTGACCCGGTCCTCGGCAGTGCGCCT-3' and (R) 5'-AGACGTGCCAGAGAAGCATTGTCAA-3'; GAPDH, (F) 5'-ACAACTCTCTCAAGATTGTCAGCAA-3' and 
(R) 5'-ACTTTGTGAAGCTCATTTCCTGG-3'. The amplification conditions were as follows: initial denaturation at $95^{\circ} \mathrm{C}$ for $15 \mathrm{~s}$, and 40 cycles at $95^{\circ} \mathrm{C}$ for $10 \mathrm{~s}, 55^{\circ} \mathrm{C}$ for $30 \mathrm{~s}$, and $72^{\circ} \mathrm{C}$ for $45 \mathrm{~s}$. Quantitative PCR was performed using a Corbett RG-6000 real-time PCR system (Corbett Life Sciences, Mortlake, Australia) according to the manufacturer's guidelines. PCR reactions for each sample were performed in triplicate. The relative expression is shown after normalization to GAPDH.

\section{Immunofluorescence}

HUVECs, grown on glass coverslips, were fixed with $4 \%$ paraformaldehyde for 20 min at $4^{\circ} \mathrm{C}$ and extracted in $0.5 \%$ Triton X-100 for $10 \mathrm{~min}$. After extensive washing in PBS, samples were incubated with primary antibodies for $1 \mathrm{~h}$ at room temperature. After three washes in PBS, cells were then incubated with secondary antibodies for an additional $1 \mathrm{~h}$. Nuclei were stained by $1 \mathrm{mg} / \mathrm{L} \mathrm{4,} \mathrm{6-diamidino-2-phenylindole}$ (DAPI, Sigma) solution for $15 \mathrm{~min}$ in the dark. Stained cells were viewed under a fluorescent microscope equipped with appropriate filters (Nikon, Japan).

Stable knockdown of vimentin by shRNA

The lentiviral vector specific for vimentin shRNA and negative control shRNA were purchased from Hanheng Biotechnology (Shanghai, China). The target sequence for vimentin shRNA was GCTCGTCACCTTCGTGAATAC. Lentiviral particles were generated by combining $10 \mu \mathrm{g}$ of shRNA lentiviral plasmid with $10 \mu \mathrm{g}$ of Lenti-HG packaging mix into 293T cells, using $60 \mu \mathrm{l}$ of HG transgene reagent. Viral supernatants were harvested at $72 \mathrm{~h}$, centrifuged at $4500 \times \mathrm{g}$ for $5 \mathrm{~min}$, filtered through a $0.45 \mu \mathrm{m}$ filter and incubated with HUVECs and polybrene $(5 \mu \mathrm{g} / \mathrm{ml})$. After $48 \mathrm{~h}$ of viral transduction, cells were given fresh growth media. Stable transfectants were selected in the presence of $0.2 \mu \mathrm{g} / \mathrm{ml}$ puromycin for 3 weeks.

\section{Statistical analysis}

All experiments were performed at least three times. Data were expressed as means \pm SD. Oneway ANOVA followed by Student's t test was used for the statistical analysis by employing SPSS program. Differences were considered statistically significant at $\mathrm{P}<0.05$.

\section{Results}

TSG inhibited oxLDL-induced endothelial cell dysfunction

First, the dose-dependent cell injury induced by oxLDL was evaluated by determining the percentage of MTT reduction. As shown in Fig. 1B, the viability of the cells exposed to 150 and $200 \mu \mathrm{g} / \mathrm{ml}$ of oxLDL for $24 \mathrm{~h}$ decreased to $76.7 \pm 4.4$ and $63.1 \pm 5.7 \%$ compared with the control, respectively. Thus, $200 \mu \mathrm{g} / \mathrm{ml}$ of oxLDL for $24 \mathrm{~h}$ was selected for further experiments in this study. To investigate the protective effects of TSG against oxLDL-induced HUVECs injury, various concentrations of TSG were added to the culture medium $12 \mathrm{~h}$ before oxLDL was added. As shown in Fig. 1B and C, there were no significant differences in cell viability in TSG-treated HUVECs compared to the control group, while TSG pretreatment with 50 and $100 \mu \mathrm{M}$ significantly attenuated the decrease in cell viability caused by oxLDL treatment and the cell viability increased to $74.2 \pm 8.8$ and $91.3 \pm 3.3 \%$, respectively. LDH assay revealed that oxLDL significantly increased the LDH release in the culture supernatant. This cytotoxic effect was also alleviated by pretreatment with 50 and $100 \mu \mathrm{M}$ TSG (Fig. 1D).

The induction of apoptosis in oxLDL-treated HUVECs was detected with Hochest 33258 staining (Fig. 2A). More apoptotic cells were observed in oxLDL-treated cultures, yet pretreatment with TSG at 50 and $100 \mu \mathrm{M}$ significantly reduced the amount of apoptotic cells in comparison to the oxLDL-treated group. OxLDL-enhanced adhesiveness of human monocytes to endothelium has been implicated in the initial stage of atherogenesis [33]. To test the effect of TSG on monocyte adhesion to HUVECs, the adhesiveness of U937 cells to oxLDL- activated HUVECs with or without TSG pretreatment was examined. As shown in Fig. 2B, oxLDL stimulated a marked increase in adherence of U937 cells to HUVECs; however, the effect was clearly reduced by TSG treatment in a dose-dependent manner. 


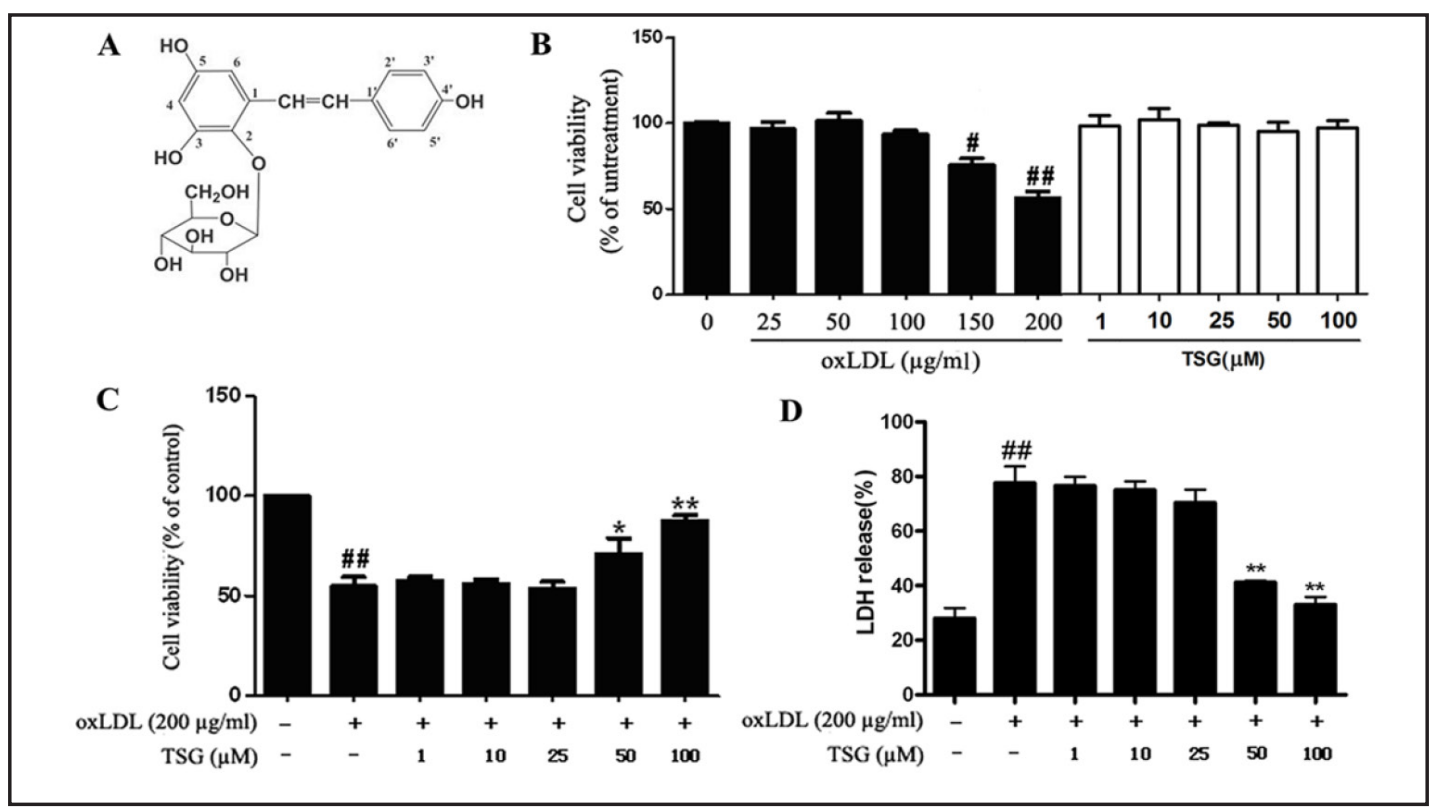

Fig. 1. Effects of TSG on oxLDL-induced cytotoxicity in human umbilical vein endothelial cells (HUVECs). (A) Chemical structure of TSG. (B) Dose-dependent effects of oxLDL and TSG on cell viability in HUVECs, as assessed by the MTT assay. (C) Effects of TSG against cytotoxicity induced by oxLDL, as assessed by the MTT assay. (D) Effects of TSG on cell death induced by oxLDL, as assessed by testing LDH activity in the supernatant culture medium. Cells without treatment of TSG and oxLDL belong to the control group. Data are expressed as means $\pm \mathrm{SD}(\mathrm{n}=3)$. \# $\mathrm{P}<0.05$ and \#\# $\mathrm{P}<0.01$ vs. the control group. ${ }^{*} \mathrm{P}<0.05$ and ${ }^{* *} \mathrm{P}<0.01$ vs. the group treated with oxLDL alone.

TSG suppressed oxLDL-induced vimentin expression and cleavage in HUVECs

Vimentin has been proved to be required for cell apopotosis and adhesion, and it is a possible molecular target responsible for atherogenesis suppression induced by TSG treatment $[14,16,24]$. Therefore, we examined the effects of TSG on vimentin expression and cleavage in oxLDL-induced HUVECs. As shown in Fig. 3A, vimentin filament assembly was disrupted by oxLDL treatment and vimentin was present in the punctate, granular aggregates (shown by arrows). Notably, pretreatment with TSG markedly inhibited oxLDLinduced vimentin disruption (Fig. 3A). Incubation of HUVECs with oxLDL enhanced vimentin expression at both the mRNA (Fig. 3C) and protein levels (Fig. 3B), whereas pretreatment with 50 or $100 \mu \mathrm{M}$ TSG abolished the induction of vimentin. Vimentin cleavage has been reported in response to various inducers of apoptosis in many cell types [23-25, 27]. Treatment of HUVECs with oxLDL resulted in the appearance of vimentin fragments. As shown in Fig. 3B, native vimentin (58 $\mathrm{kDa}$ ) was cleaved into five fragments $(54,50,42,36$ and $30 \mathrm{kDa})$ after oxLDL treatment. Degradation of vimentin was significantly inhibited by pretreatment of 50 or $100 \mu \mathrm{M}$ TSG and no cleavage of vimentin was detected in the cells after pretreatment with $100 \mu \mathrm{M}$ TSG.

TSG'S inhibition of vimentin expression might be involved in TGFß1/Smad pathway in HUVECS

Vimentin expression was reported to be regulated by TGF $\beta 1 /$ Smad signaling pathway in the skeletal myogenic cell line [28]. We further analyzed the effects of TSG on the expression of TGF $\beta 1$ and the phosphorylation of Smad 2 and 3 by Western blot analysis in oxLDL-induced HUVECs. As illustrated in Fig. 4A and B, cells induced by oxLDL showed an increased level of TGF $\beta 1$, phospho-Smad2 and 3 compared with the control. Pretreatment with 50 or 100 $\mu \mathrm{M}$ TSG effectively attenuated the oxLDL-induced TGF $\beta 1$ expression and Smads activation via phosphorylation. TGF $\beta 1$ receptors phosphorylate Smad2 and/or Smad3, which in turn 


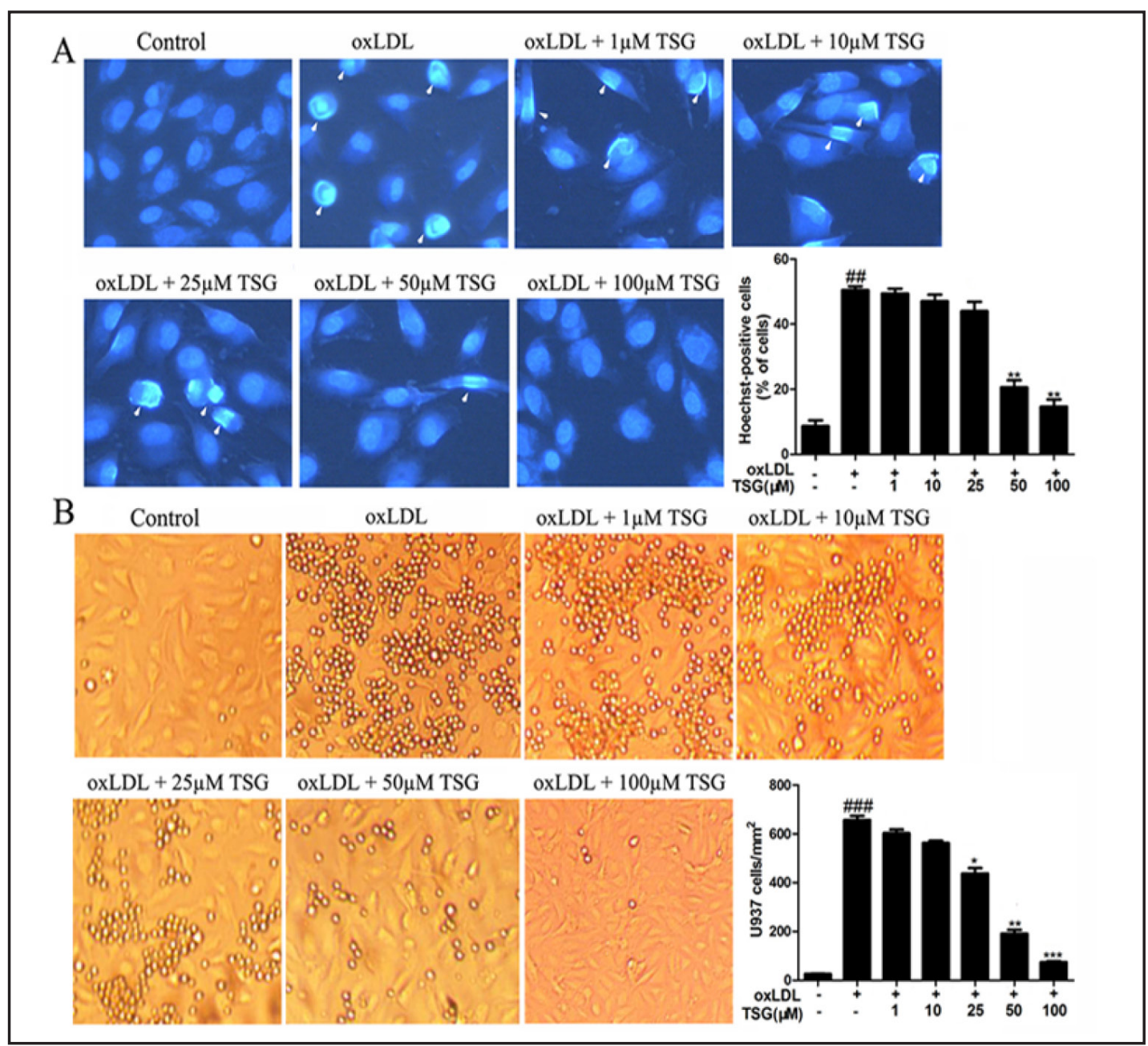

Fig. 2. Effects of TSG on oxLDL-induced apoptosis and monocyte adhesion in HUVECs. Cells were pretreated with TSG $(1,10,25,50$, and $100 \mu \mathrm{M})$ for $12 \mathrm{~h}$ before incubating with $200 \mu \mathrm{g} / \mathrm{ml}$ oxLDL for $24 \mathrm{~h}$. (A) Cells were stained by Hoechst 33258 and observed under fluorescence microscopy (at a magnification of $200 \times$ ). White arrows indicate cell apoptosis. Histogram shows the ratio of Hoechst-positive cells. (B) Representative photomicrographs of U937 monocytic cells adherent to HUVECs are shown. Quantification is expressed as number of adherent $U 937$ cells per $\mathrm{mm}^{2}$. Data are expressed as means $\pm \mathrm{SD}(\mathrm{n}=3)$. \#\# $\mathrm{P}<0.01$ and \#\#\# $\mathrm{P}<0.001$ vs. the control group. ${ }^{*} \mathrm{P}<0.05$, ${ }^{* *} \mathrm{P}<0.01$ and ${ }^{* * *} \mathrm{P}<0.001$ vs. the oxLDL group.

bind to Smad4 to induce translocation into the nucleus [34]. To examine whether TSG influences the nuclear translocation of the Smad complex, Smad4 was analyzed by direct immunofluorescence assay. As revealed in Fig. 4C, Smad4 immunoreactivity in the control group was found in the cytoplasm, but little in the nuclei. Exposure to oxLDL caused the majority of intracellular Smad4 translocation from the cytoplasm to the nucleus, as shown by strong Smad4 staining in the nucleus. This translocation was greatly inhibited by pretreatment with 50 or $100 \mu \mathrm{M}$ TSG.

\section{TSG suppressed oxLDL-induced caspase-3 activation in HUVECS}

Caspases, especially caspase-3, have been implicated in fragmentation of vimentin during cell apoptosis $[25,26]$. To confirm the involvement of caspase- 3 in vimentin proteolysis in oxLDL-induced apoptosis and whether TSG inhibited vimentin cleavage by suppressing caspase- 3 activation, we determined the activity of caspase- 3 by Western blot and colorimetric assay. After incubation with oxLDL for $24 \mathrm{~h}$, caspase-3 was proteolytically cleaved into p20 and p17 subunits to become an active enzyme (Fig. 5A). Colorimetric 
Fig. 3. Inhibition of TSG on vimentin expression and cleavage induced by oxLDL in HUVECs. Cells were pretreated with TSG for $12 \mathrm{~h}$ before incubating with $200 \mu \mathrm{g} / \mathrm{ml}$ oxLDL for $24 \mathrm{~h}$. Cells without any treatment belong to the control group. (A) Immunofluorescence of vimentin filaments (red) and nuclei stained with DAPI (blue) (magnification $200 \times$ ). Cells were treated with $100 \mu \mathrm{M}$ TSG before incubating with oxLDL. oxLDL induces the rearrangement and cleavage of vimentin intermediate filaments into punctate, granular aggregates (indicated by arrows). TSG blocks oxLDL-induced vimentin disruption. (B) Western blot analysis of vimentin fragmentation. Vimentin is rapidly proteolyzed during oxLDL into multiple fragments. (C) Real-time RT-PCR analysis for vimentin. Histogram shows the ratios of vimentin mRNA to GAPDH. Data are expressed as means $\pm \operatorname{SD}(n=3)$. \#\# $\mathrm{P}<0.01$ vs. the control group. ${ }^{*} \mathrm{P}<0.05$ and ** $\mathrm{P}<0.01$ vs. the group treated with oxLDL alone.

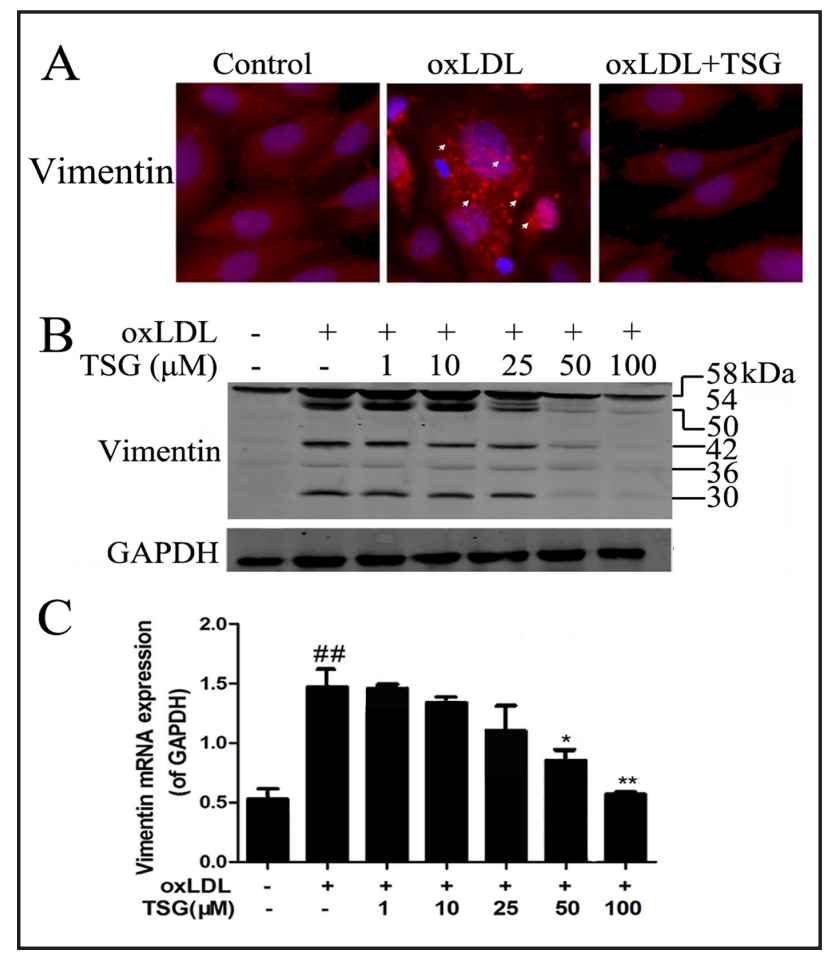

assay also revealed that oxLDL significantly upregulated the activity of caspase-3 (Fig. 5B). Pretreatment with TSG at 50 and $100 \mu \mathrm{M}$ markedly suppressed the cleavage and the activity of caspase-3, which demonstrates that TSG has an inhibitory effect on caspase-3 activation and vimentin proteolysis.

TSG inhibited oxLDL-induced expression of adhesion molecules and colocalization with vimentin

The effects of TSG on the expression of ICAM-1 and VCAM- 1 in HUVECs exposed to oxLDL were subsequently examined. As shown in Fig. 6A, the expression of ICAM-1 and VCAM-1 were significantly higher in HUVECs that had been treated with oxLDL than in the control cells. This induction of adhesion molecules expression was significantly ameliorated by the presence of TSG. Vimentin was reported to associate with the expression and the distribution of ICAM-1 and VCAM-1 [16]. Using fluorescent microscope we analyzed the effects of TSG on colocalization of vimentin with adhesion molecules. A colocalization of vimentin with ICAM-1 or VCAM-1 was detected in oxLDL-induced HUVECs, but not in controls and HUVECs pretreated with $100 \mu \mathrm{M}$ TSG (Fig. 6B and C). We conclude that TSG inhibits oxLDL-induced colocalization of vimentin with adhesion molecules.

shRNA mediated suppression of vimentin inhibited oxLDL-stimulated endothelial cell apoptosis and adherence of U937 cells to HUVECS

To illustrate whether vimentin is responsible for oxLDL-induced endothelial cell dysfunction, vimentin expression was suppressed by shRNA and the potential functional consequences of vimentin suppression on endothelial cell apoptosis and adhesiveness were analyzed. After having demonstrated successful suppression after shRNA transduction (Fig. 7A), TUNEL staining analyses revealed that vimentin suppression significantly reduced oxLDL-induced endothelial cell apoptosis and the number of TUNEL-positive cells decreased to $15.1 \pm 2.6 \%$ compared with the control shRNA group (Fig. 7B). The adhesion between HUVECs and U937 cells induced by oxLDL was markedly impaired in vimentin-suppressed HUVECs, when compared with the control shRNA group after oxLDL treatment (Fig. 7C). 


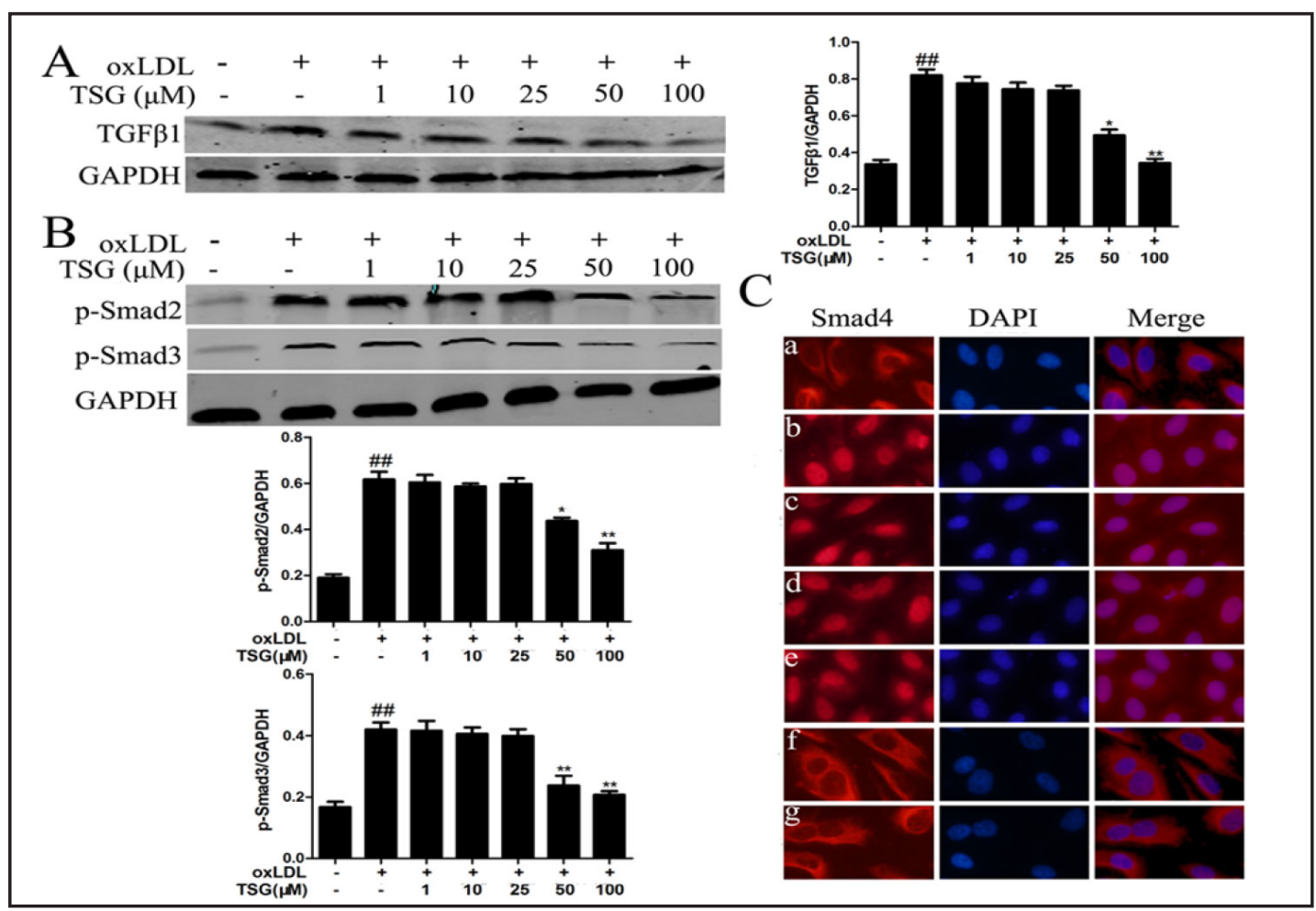

Fig. 4. TSG inhibits TGF $\beta 1 /$ Smad signaling pathway induced by oxLDL in HUVECs. Cells were pretreated with TSG $(1,10,25,50$, and $100 \mu \mathrm{M})$ for $12 \mathrm{~h}$ before incubating with $200 \mu \mathrm{g} / \mathrm{ml}$ oxLDL for $24 \mathrm{~h}$. (A) Western blot analysis of TGF $\beta 1$. The protein levels were quantified by densitometric analysis, normalized to GAPDH. (B) Western blot analysis of phospho-Smads $(-2,-3)$. The levels of $p$-Smads were quantified and normalized to GAPDH. (C) Effects of TSG on nuclear translocation of Smad4 in oxLDL-induced HUVECs (magnification $200 \times$ ). (a) control group (without treatment), (b) oxLDL-induced group, (c) oxLDL group pretreated with 1 $\mu \mathrm{M}$ TSG, (d) oxLDL group pretreated with $10 \mu \mathrm{M}$ TSG, (e) oxLDL group pretreated with $25 \mu \mathrm{M}$ TSG, (f) oxLDL group pretreated with $50 \mu \mathrm{M}$ TSG, (g) oxLDL group pretreated with $100 \mu \mathrm{M}$ TSG. (Left) Smad4 was detected by Cy3-labeled immunostaining (red); (middle) nuclei were stained by DAPI (blue); (right) merged images. Data are expressed as means \pm SD $(n=3)$. \#\# $\mathrm{P}<0.01$ vs. the control group. ${ }^{*} \mathrm{P}<0.05$ and ${ }^{* *} \mathrm{P}<0.01$ vs. the group treated with oxLDL alone.

Fig. 5. Inhibitory effects of TSG on oxLDL-induced caspase- 3 activation. Cells were pretreated with TSG $(1,10,25,50$, and $100 \mu \mathrm{M})$ for $12 \mathrm{~h}$ before incubating with $200 \mu \mathrm{g} / \mathrm{ml}$ oxLDL for $24 \mathrm{~h}$. (A) Western blot analysis of caspase-3 cleavage. (B) Caspase-3 activity detected by colorimetric assay. Data are expressed as means $\pm \mathrm{SD}(\mathrm{n}=3)$. \#\#\# $\mathrm{P}<0.001$ vs. the control group. ${ }^{* *} \mathrm{P}<0.01$ and ${ }^{* * *} \mathrm{P}<0.001$ vs. the group treated with oxLDL alone.

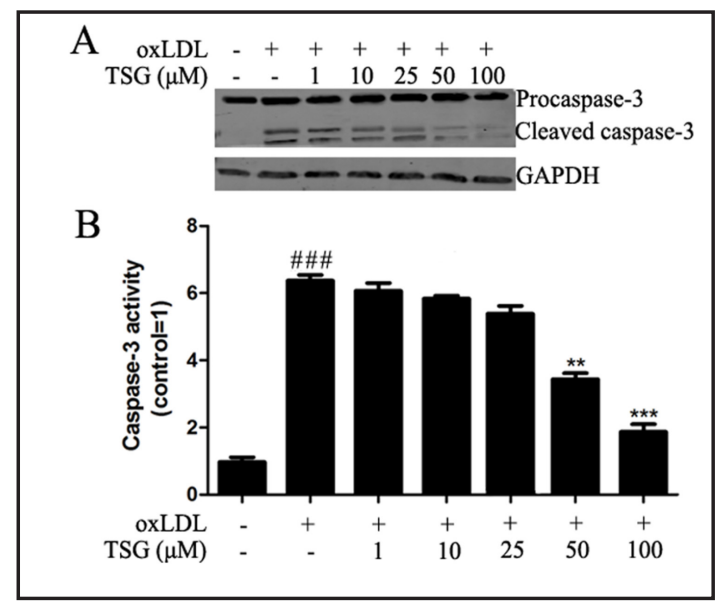

\section{Discussion}

Endothelial injury caused by ox-LDL is commonly considered as an early indication of atherosclerosis followed by endothelial cell apoptosis and leukocyte adhesion $[2,3,5]$. 
Fig. 6. Western blot analysis and Immunofluorescence of adhesion molecules and vimentin. (A) Western-blot detection of VCAM-1 and ICAM-1. HUVECs were pretreated with TSG $(1,10,25$, 50 , and $100 \mu \mathrm{M}$ ) for $12 \mathrm{~h}$ before incubating with $200 \mu \mathrm{g} / \mathrm{ml}$ oxLDL for $24 \mathrm{~h}$. (B) Immunofluorescence images (magnification $200 \times$ ) of vimentin (green) and ICAM-1 (red) as double staining (merge). Nuclei were stained by DAPI (blue). Cells were treated with $100 \mu \mathrm{M}$ TSG before incubating with $200 \mu \mathrm{g} / \mathrm{ml}$ oxLDL. TSG inhibits oxLDL-induced colocalization of vimentin with ICAM-1. (C) Immunofluorescence images (magnification $200 \times$ ) of vimentin (green) and VCAM-1 (red) as double staining (merge). Nuclei were stained by DAPI (blue). Cells were treated with $100 \mu \mathrm{M}$ TSG before incubating with $200 \mu \mathrm{g} / \mathrm{ml}$ oxLDL. TSG inhibits oxLDL-induced colocalization of vimentin with VCAM-1.

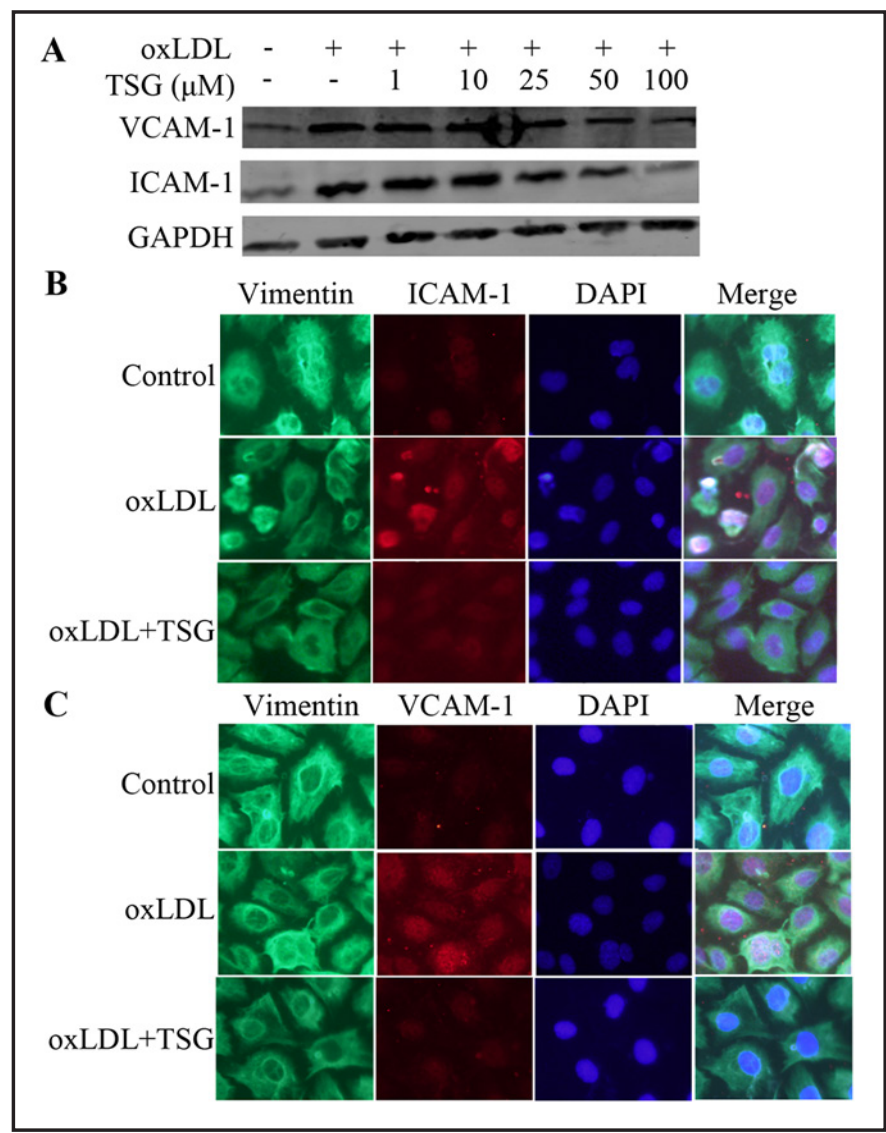

As the bioactive and marker component of $P$. multiflorum, TSG has been proved to exhibit significant anti-atherosclerotic activity in rats $[8,9]$. However, whether TSG could inhibit oxLDL-induced endothelial injury has not been studied. In this study, MTT assay showed that pretreatment with TSG could effectively suppress the reduced cell viability induced by oxLDL. LDH releasing assessment showed that TSG could significantly inhibit oxLDL-induced LDH release. Furthermore, nuclear condensation and monocyte adhesion to HUVECs were greatly inhibited in oxLDL-treated cells with TSG pretreatment. These data suggested that TSG significantly protected HUVECs against oxLDL-induced injury.

To understand why TSG could protect HUVECs, we next investigated the underlying mechanism(s). Vimentin has been proved to be one of the key proteins in TSG treatment for atherosclerosis in rats [14]. We therefore investigated vimentin expression regulated by TSG in oxLDL-induced HUVECs. Our results showed that vimentin protein expression was inhibited by TSG pretreatment in oxLDL-induced HUVECs. In order to demonstrate whether vimentin expression is responsible for oxLDL-induced endothelial injury, we induced a knockdown of vimentin expression using shRNA technology and then investigated cell apoptosis and monocyte adhesion in HUVECs induced by oxLDL after vimentin suppression. Remarkably, in vimentin-suppressed HUVECs the oxLDL-induced apoptosis and monocyte adhesion were significantly smaller than those observed in the control shRNA group. These results suggested that TSG's protection against oxLDL-induced endothelial injury is caused, at least in part, by its inhibition of vimentin expression. Vimentin expression is often upregulated in response to TGF $\beta$ in primary osteoblasts and osteoblastic cell lines or during EMT and cancer progression $[35,36]$. It has been reported that TGF $\beta 1$ stimulates vimentin expression through Smad signaling in the skeletal myogenic cell line [28]. We showed that TSG decreased TGF $\beta 1$ expression, as well as phosphorylation of Smad2 and Smad3 in oxLDL-activated HUVECs. In addition, we found that TSG pretreatment markedly prevented oxLDL-induced nuclear translocation of Smad4. Therefore, our results suggested that TSG's 


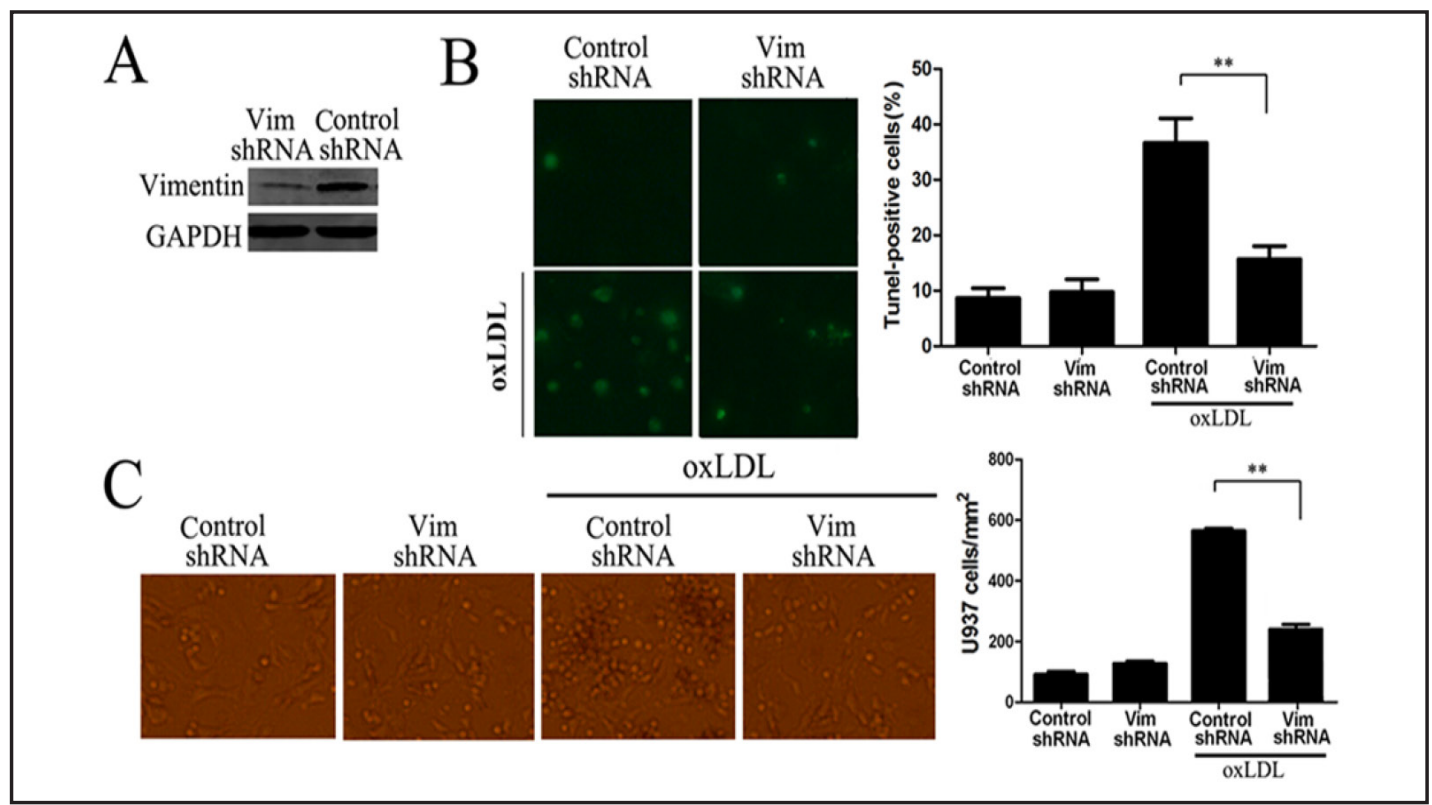

Fig. 7. (A) Detection of vimentin suppression via Western blot. Control shRNA: HUVECs transduced with lentiviruses delivering control shRNA. Vim shRNA: HUVECs transduced with lentiviruses delivering vimentin shRNA. Stable cell lines were selected with $0.2 \mu \mathrm{g} / \mathrm{ml}$ puromycin for 3 weeks. (B) Cell apoptosis detected by TUNEL assay (magnification $200 \times$ ). HUVECs transduced with shRNA were treated with $200 \mu \mathrm{g} / \mathrm{ml}$ oxLDL for $24 \mathrm{~h}$. Histogram shows the ratio of TUNEL-positive cells. Data are expressed as means \pm SD $(n=3)$. ** $\mathrm{P}<0.01$ vs. control shRNA group treated with oxLDL. (C) Adhesion assay of U937 monocytes to HUVECs transduced with shRNA. HUVECs transduced with shRNA were treated with $200 \mu \mathrm{g} / \mathrm{ml}$ oxLDL for $24 \mathrm{~h}$. Histogram shows adherent U937 cells per $\mathrm{mm}^{2}$. Data are expressed as means $\pm \mathrm{SD}(\mathrm{n}=3) .{ }^{* *} \mathrm{P}<0.01$ vs. control shRNA group treated with oxLDL.

Fig. 8. Schematic diagram showing cytoprotective signaling of TSG in oxLDL-induced endothelial dysfunction. An arrowhead indicates activation or induction, and a vertical bar indicates inhibition or blockade.

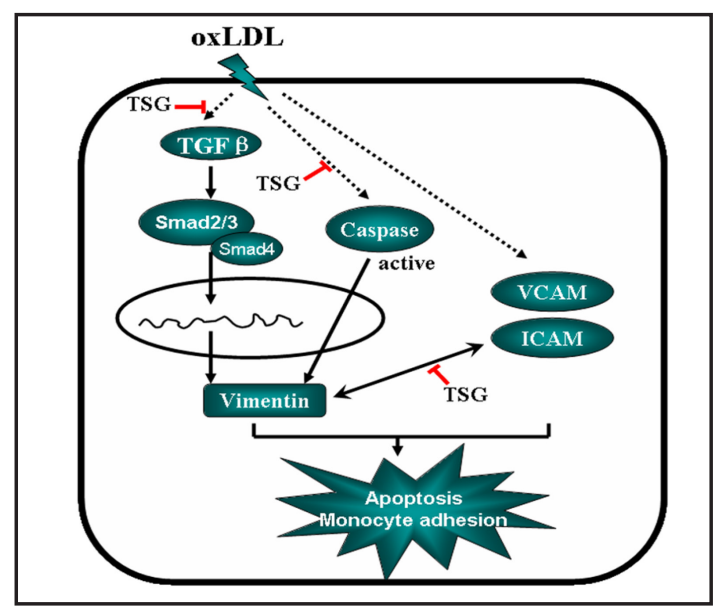

inhibition of vimentin is mediated by its down-regulation of TGF $\beta /$ Smad signaling pathway in oxLDL-induced HUVECs.

It is generally known that vimentin is specific to the apoptotic process and rapidly degraded during apoptosis by multiple caspases, resulting in diverse vimentin fragments $[24,26]$. Our study found that vimentin filament assembly was redistributed during oxLDLinduced apoptosis and present in the punctate, granular aggregates. Western blot analysis showed that native vimentin was cleaved into different fragments with different molecular weights after oxLDL treatment in HUVECs. Notably, this redistribution and cleavage of vimentin induced by oxLDL were inhibited by TSG pretreatment. Caspases, especially 
caspase-3, have been implicated in fragmentation of vimentin during cell apoptosis [25, 26]. We showed that TSG reduced the activity of caspase-3 in oxLDL-treated HUVECs. The mechanisms by which TSG protects HUVECs against the apoptotic effects of oxLDL could be in part by inhibiting caspase- 3 activation and subsequent vimentin fragmentation.

ICAM-1 and VCAM-1 are considered to play key roles at the early stage of inflammatory response to facilitate leukocytes adhesion and transmigration in vascular endothelial cells. We observed that TSG inhibited the expression of ICAM-1 and VCAM-1 in oxLDL-activated HUVECs. Vimentin has been reported to participate in lymphocyte adhesion and the formation of an anchoring structure for the involved adhesion molecules [16]. In this study, our data demonstrated that TSG suppressed the colocalization of vimentin with adhesion molecules, suggesting that TSG reduction of monocytes adhesion is caused, at least in part, by downregulating the expression of adhesion molecules and the colocalization of vimentin with ICAM-1 or VCAM-1.

\section{Conclusions}

Taken together, our data provide for the first time the direct evidence for the inhibitory effect of TSG on oxLDL-induced endothelial cell dysfunction. These protective effects may be mediated in part by inhibiting vimentin expression and cleavage via interruption of the TGF $\beta /$ Smad signaling pathway and caspase- 3 activation; suppressing the expression of adhesion molecules and their colocalization with vimentin (as shown in Fig. 8).

\section{Disclosure Statement}

The authors had no conflicts of interest to declare in relation to this article.

\section{Acknowledgements}

This work was supported by a grant from the National Natural Sciences Foundation of China (No. 81202879), the Natural Science Foundation of Jiangsu Province (No. BK20130395), the National Natures Science Foundation of Nantong City (No. BK2012087) and the Priority Academic Program Development of Jiangsu Higher Education Institutions.

\section{References}

1 Libby P: Inflammation in atherosclerosis. Nature 2002;420:868-874.

-2 Liu S, Shen H, Xu M, Liu O, Zhao L, Guo Z, Du J: FRP inhibits ox-LDL-induced endothelial cell apoptosis through an Akt-NF-\{kappa\}B-Bcl-2 pathway and inhibits endothelial cell apoptosis in an apoE-knockout mouse model. Am J Physiol Endocrinol Metab 2010;299:E351-363.

-3 Chen B, Wang W, Shen T, Qi R: Thioredoxin1 downregulates oxidized low-density lipoprotein-induced adhesion molecule expression via Smad3 protein. PLoS One 2013;8:e76226.

4 Dimmeler S, Haendeler J, Galle J, Zeiher AM: Oxidized low-density lipoprotein induces apoptosis of human endothelial cells by activation of CPP32-like proteases. A mechanistic clue to the 'response to injury' hypothesis. Circulation 1997;95:1760-1763.

-5 Ou HC, Song TY, Yeh YC, Huang CY, Yang SF, Chiu TH, Tsai KL, Chen KL, Wu YJ, Tsai CS, Chang LY, Kuo WW, Lee SD: EGCG protects against oxidized LDL-induced endothelial dysfunction by inhibiting LOX-1-mediated signaling. J Appl Physiol (1985) 2010;108:1745-1756. 
6 Wang X, Zhao L, Han T, Chen S, Wang J: Protective effects of 2,3,5,4'-tetrahydroxystilbene-2-0-beta-dglucoside, an active component of Polygonum multiflorum Thunb, on experimental colitis in mice. Eur J Pharmacol 2008;578:339-348.

7 Wang T, Gu J, Wu PF, Wang F, Xiong Z, Yang YJ, Wu WN, Dong LD, Chen JG: Protection by tetrahydroxystilbene glucoside against cerebral ischemia: involvement of JNK, SIRT1, and NF-kappaB pathways and inhibition of intracellular ROS/RNS generation. Free Radic Biol Med 2009;47:229-240.

-8 Zhang W, Wang CH, Li F, Zhu WZ: 2,3,4',5-Tetrahydroxystilbene-2-O-beta-D-glucoside suppresses matrix metalloproteinase expression and inflammation in atherosclerotic rats. Clin Exp Pharmacol Physiol 2008;35:310-316.

-9 Zhang W, Xu XL, Wang YQ Wang CH, Zhu WZ: Effects of 2,3,4',5-tetrahydroxystilbene 2-O-beta-D-glucoside on vascular endothelial dysfunction in atherogenic-diet rats. Planta Med 2009;75:1209-1214.

10 Han X, Ling S, Gan W, Sun L, Duan J, Xu JW: 2,3,5,4'-tetrahydroxystilbene-2-0-beta-d-glucoside ameliorates vascular senescence and improves blood flow involving a mechanism of p53 deacetylation. Atherosclerosis 2012;225:76-82.

11 Liu QL, Xiao JH, Ma R, Ban Y, Wang JL: Effect of 2,3,5,4'-tetrahydroxystilbene-2-O-beta-D-glucoside on lipoprotein oxidation and proliferation of coronary arterial smooth cells. J Asian Nat Prod Res 2007;9:689697.

12 Xu XL, Huang YJ, Wang YQ Chen XF, Zhang W: 2,3,4',5-Tetrahydroxystilbene-2-0-beta-d-glucoside inhibits platelet-derived growth factor-induced proliferation of vascular smooth muscle cells by regulating the cell cycle. Clin Exp Pharmacol Physiol 2011;38:307-313.

13 Xu XL, Huang YJ, Chen XF, Lin DY, Zhang W: 2,3,4',5-tetrahydroxystilbene-2-0-beta-D-glucoside inhibits proliferation of vascular smooth muscle cells: involvement of NO/cGMP/PKG pathway. Phytother Res 2012;26:1068-1074.

14 Yao W, Fan W, Huang C, Zhong H, Chen X, Zhang W: Proteomic analysis for anti-atherosclerotic effect of tetrahydroxystilbene glucoside in rats. Biomed Pharmacother 2013;67:140-145.

15 Kang JH, Kim HT, Choi MS, Lee WH, Huh TL, Park YB, Moon BJ, Kwon OS: Proteome analysis of human monocytic THP-1 cells primed with oxidized low-density lipoproteins. Proteomics 2006;6:1261-1273.

-16 Nieminen M, Henttinen T, Merinen M, Marttila-Ichihara F, Eriksson JE, Jalkanen S: Vimentin function in lymphocyte adhesion and transcellular migration. Nat Cell Biol 2006;8:156-162.

17 Qin H, Ishiwata T, Wang R, Kudo M, Yokoyama M, Naito Z, Asano G: Effects of extracellular matrix on phenotype modulation and MAPK transduction of rat aortic smooth muscle cells in vitro. Exp Mol Pathol 2000;69:79-90.

18 Lund N, Henrion D, Tiede P, Ziche M, Schunkert H, Ito WD: Vimentin expression influences flow dependent VASP phosphorylation and regulates cell migration and proliferation. Biochem Biophys Res Commun 2010;395:401-406.

19 McInroy L, Maatta A: Down-regulation of vimentin expression inhibits carcinoma cell migration and adhesion. Biochem Biophys Res Commun 2007;360:109-114.

20 Pan TL, Wang PW, Huang CC, Yeh CT, Hu TH, Yu JS: Network analysis and proteomic identification of vimentin as a key regulator associated with invasion and metastasis in human hepatocellular carcinoma cells. J Proteomics 2012;75:4676-4692.

21 Tsuruta D, Jones JC: The vimentin cytoskeleton regulates focal contact size and adhesion of endothelial cells subjected to shear stress. J Cell Sci 2003;116:4977-4984.

22 Carman CV, Springer TA: A transmigratory cup in leukocyte diapedesis both through individual vascular endothelial cells and between them. J Cell Biol 2004;167:377-388.

23 Belichenko I, Morishima N, Separovic D: Caspase-resistant vimentin suppresses apoptosis after photodynamic treatment with a silicon phthalocyanine in Jurkat cells. Arch Biochem Biophys 2001;390:5763.

24 Byun Y, Chen F, Chang R, Trivedi M, Green KJ, Cryns VL: Caspase cleavage of vimentin disrupts intermediate filaments and promotes apoptosis. Cell Death Differ 2001;8:443-450.

25 Hashimoto M, Inoue S, Ogawa S, Conrad C, Muramatsu M, Shackelford D, Masliah E: Rapid fragmentation of vimentin in human skin fibroblasts exposed to tamoxifen: a possible involvement of caspase-3. Biochem Biophys Res Commun 1998; 247: 401-406.

26 Morishima N: Changes in nuclear morphology during apoptosis correlate with vimentin cleavage by different caspases located either upstream or downstream of Bcl-2 action. Genes Cells 1999;4:401-414. 
27 Prasad SC, Thraves PJ, Kuettel MR, Srinivasarao GY, Dritschilo A, Soldatenkov VA: Apoptosis-associated proteolysis of vimentin in human prostate epithelial tumor cells. Biochem Biophys Res Commun 1998;249:332-338.

28 Wu Y, Zhang X, Salmon M, Lin X, Zehner ZE: TGFbeta1 regulation of vimentin gene expression during differentiation of the C2C12 skeletal myogenic cell line requires Smads, AP-1 and Sp1 family members. Biochim Biophys Acta 2007;1773:427-439.

29 Chung BH, Wilkinson T, Geer JC, Segrest JP: Preparative and quantitative isolation of plasma lipoproteins: rapid, single discontinuous density gradient ultracentrifugation in a vertical rotor. J Lipid Res 1980;21:284291.

-30 Price P, McMillan TJ: Use of the tetrazolium assay in measuring the response of human tumor cells to ionizing radiation. Cancer Res 1990;50:1392-1396.

- 31 Kim CS, Kim YR, Naqvi A, Kumar S, Hoffman TA, Jung SB, Kumar A, Jeon BH, McNamara DM, Irani K: Homocysteine promotes human endothelial cell dysfunction via site-specific epigenetic regulation of p66shc. Cardiovasc Res 2011;92:466-475.

-32 Kim YR, Kim CS, Naqvi A, Kumar A, Kumar S, Hoffman TA, Irani K: Epigenetic upregulation of p66shc mediates low-density lipoprotein cholesterol-induced endothelial cell dysfunction. Am J Physiol Heart Circ Physiol 2012;303:H189-196.

33 Kim JA, Territo MC, Wayner E, Carlos TM, Parhami F, Smith CW, Haberland ME, Fogelman AM, Berliner JA: Partial characterization of leukocyte binding molecules on endothelial cells induced by minimally oxidized LDL. Arterioscler Thromb 1994;14:427-433.

34 Abdollah S, Macias-Silva M, Tsukazaki T, Hayashi H, Attisano L, Wrana JL: TbetaRI phosphorylation of Smad2 on Ser465 and Ser467 is required for Smad2-Smad4 complex formation and signaling. J Biol Chem 1997;272:27678-27685.

-35 Kokkinos MI, Wafai R, Wong MK, Newgreen DF, Thompson EW, Waltham M: Vimentin and epithelialmesenchymal transition in human breast cancer--observations in vitro and in vivo. Cells Tissues Organs 2007;185:191-203.

-36 Steinert PM, Roop DR: Molecular and cellular biology of intermediate filaments. Annu Rev Biochem 1988;57:593-625. 